\title{
Modelling of butylated hydroxytoluene (BHT) transport in water in PE-HD pipe including chemical reaction of BHT oxidation
}

\author{
Anna Musz-Pomorska ${ }^{1,}$, Beata Kowalska ${ }^{1}$, and Marcin K. Widomski ${ }^{1}$ \\ ${ }^{1}$ Faculty of Environmental Engineering, Lublin University of Technology, ul. Nadbystrzycka 40B, \\ 20-618 Lublin,
}

\begin{abstract}
This paper presents laboratory and modelling studies of antioxidant butylated hydroxytoluene leaching from a water supply PE-HD pipe for laminar and turbulent flow. The reaction of BHT oxidation to BHT-OH was included in our calculations. Required input data, initial and boundary conditions were based on laboratory measurements performed for a new PE-HD pipe. Laboratory measurements covered tests of BHT content and homogeneity in pipe material as well as measurements of BHT concentration in water flowing inside the pipe loop with different velocities. The BHT content of the PE-HD pipe and its concentration in water were determined by GC-MS method. Modelling calculations of BHT concentration in water for two different types of flow were performed using the commercial CDF software Fluent (Ansys Inc.). Nonhomogeneous BHT distribution in pipe material and leaching of the antioxidant to the water were observed during laboratory tests. Numerical prediction of BHT concentration in water showed acceptable agreement between measured and calculated data. Nonetheless, the universality of the developed model is limited by the great diversity of available plastic pipe materials with various contents and technological amendments.
\end{abstract}

\section{Introduction}

The quality of drinking water received by customers results from the initial qualitative characteristics of water and physical, chemical and biological processes occurring during water flow through the water network pipelines. These processes, related also to pipe materials, may influence the deterioration of water quality in water supply networks and inhouse installations [1]. In the case of polymer materials, the presence of additional compounds such as antioxidants, stabilizers or pigments in the structure of the polymer creates the risk of their migration to water [1-4].

Studies presented by Anselme et al. [2] showed that the antioxidant butylated hydroxytoluene (BHT), commonly applied in the plastics industry, including pipe production, is responsible for odour of water transported through pipelines made of PE. The presence of BHT in water after migration tests for various plastic materials, including

* Corresponding author: A.Musz@,wis.pol.lublin.pl 
polymer pipelines, was reported by Gandek et al. [5], and Skjevrak et al. [1]. The presence of BHT in water during its flow through the plastic pipeline was reported by Widomski et al. $[6,7]$ as well as by Musz-Pomorska and Kowalska [8].

It was reported that BHT metabolization may occur as a result of oxidation of alkyl substituent and aromatic rings [9]. The main product of BHT degradation caused by alkyl substituent oxidation is BHT-OOH (3,5-di-t-butyl-4-hydroxybenzoic acid), which can be created by the accompanying alcohol BHT-OH (2,6-di-t-butyl-4-(hydroxymethyl)phenol) or aldehyde BHT-CHO (3,5-di-t-butyl-4-hydroxybenzaldehyde). The further oxidation results in creation of, inter alia, BHT-Q (2,6-di-t-butylcyclohexa-2,5-diene-1,4-dione) [9, 10].

There are several known attempts to numerically model the organic compounds' migration from plastic pipes to water, under static and dynamic conditions. For instance, Denberg et al. [3] presented the modelled prediction of the maximum possible concentration of the antioxidant Irganox 1010 for various types of water flow inside a PE pipeline. The model of stabilizers released to water from PVC pipe, including dimethyl tin and dibutyl tin, was presented by Adams et al. [11], while BHT release to water and its transport inside the pipeline was presented by Widomski et al. [6, 7]. However, in the previous work reported by Widomski et al. [6, 7], the modelling results for BHT migration and propagation in flowing water with transient flow inside the PE 100 pipeline showed visible overestimation of BHT concentration in water during the experiment. It was also suggested that reactions of BHT degradation should be included in numerical modelling to obtain a better fit of the modelled and measured data.

The aim of our study presented in this paper was the computational assessment of BHT leaching to water in variable flow conditions. BHT was selected for our research as one of the typical antioxidants applied to PE pipe production and for leaching to water from pipe material. Our modelling attempt covered simulations of transport of BHT released to water from the tested PE-HD pipe in conditions of laminar and turbulent flow. The reaction of BHT oxidation influenced by oxygen present in water was included in our model. The commercial software Fluent (Ansys Inc.) based on computational fluid dynamics (CFD) was used in our numerical studies.

\section{Materials and methods}

\subsection{Material}

The brand new $32 \times 3.0 \mathrm{~mm}$ PE-HD 80 pipes, produced in Poland according to PN-EN 12201-2:2011 [12], were used in our studies. The pipes were purchased directly from the producer. The basic properties of PE material used in our researches were as follows:

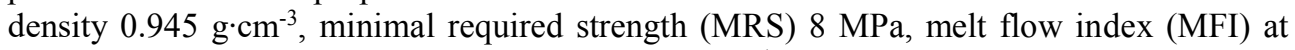
temperature of $190^{\circ} \mathrm{C}$ and load of $2 \mathrm{~kg}, 17 \mathrm{~g} \cdot(10 \mathrm{~min})^{-1}$.

\subsection{Laboratory setup}

The closed-loop pipeline laboratory setup, presented in Fig. 1, utilizing $30 \mathrm{~m}$ of PE-HD pipe was used to determine the content of BHT in flowing water. The inner area of the applied pipe was equal to $2.45 \mathrm{~m}^{2}$, while the volume of water body inside the setup was $15.9 \mathrm{dm}^{3}$.

Before beginning of the qualitative tests, the laboratory setup was rinsed with a velocity of $1.0 \mathrm{~m} \cdot \mathrm{s}^{-1}$ by deionized water prepared by Milli-Q Millipore (Molsheim, Germany) apparatus. Then, the installation was filled with the deionized water applied to our tests. The basic characteristics of the deionized water used were as follows: TOC $\leq 0.5 \mu \mathrm{g} \cdot \mathrm{dm}^{-3}$ and resistivity 
18.2 $\mathrm{M} \Omega \mathrm{m}$. The constant mean velocity of flow for each applied series of measurements was sustained by the centrifugal pump installed in the regulator of rotational speed.

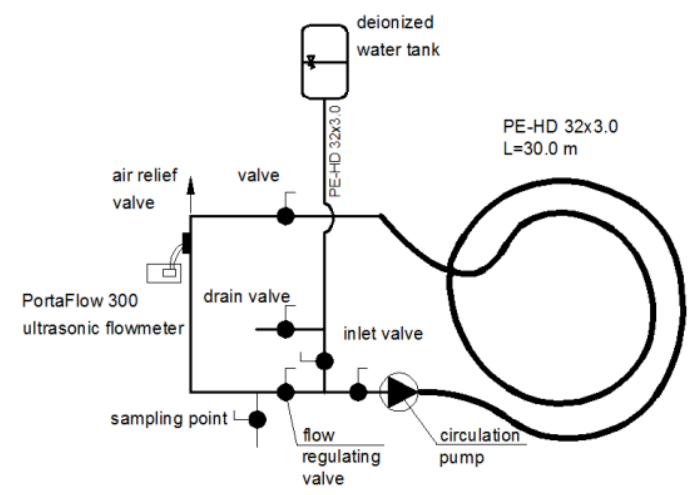

Fig. 1. Scheme of laboratory setup.

\subsection{Material analysis - homogeneity of BHT in PE-HD pipe material}

Three pieces of PE-HD pipe were tested to determine the homogeneity of BHT in the material: a brand new one and two samples which were used as the main components of the laboratory setup (Fig. 1). The each sampled part of the studied pipe, $5 \mathrm{~cm}$ in length and with mass equal to approx. 13 grams, was sliced into approx. 2.5 gram layers representing the full cross-section of the pipe. Overall, 15 samples of pipe material were extracted in tetrahydrofuran, according to the methodology by Bertoldo and Ciardelli [13]. The process of purification was performed by high resolution gel chromatography (HR-SEC) in an HPLC liquid chromatography (Waters Breeze system) 1525 Binary HPLC Pump, equipped with Waters 2487 Dual $\lambda$ Absorbance Detector. Then, the extract was analysed by Trace GC Ultra with mass detector MS Polaris Q Ion Trap by Thermo, US (GC-MS) method, according to the already published methodology [14].

\subsection{Water analysis}

\subsubsection{Measurements of BHT migration to water}

The studies concerning BHT migration to water under dynamic conditions of flow were performed in the laboratory setup presented in Fig. 1. Two different applied velocities of water flow reflecting the conditions of laminar $\left(\mathrm{v}=0.08 \mathrm{~m} \cdot \mathrm{s}^{-1}\right.$, Reynolds number $\mathrm{Re}=1733)$ and turbulent $\left(\mathrm{v}=2.5 \mathrm{~m} \cdot \mathrm{s}^{-1}, \mathrm{Re}=54816\right)$ flow were applied. The time duration of the experiment was $147 \mathrm{hr}$. The constant temperature of water inside the closed-loop installation equal to $25^{\circ} \mathrm{C} \pm 5^{\circ} \mathrm{C}$ was sustained during the laboratory test.

Water samples for qualitative measurements were sampled from the laboratory installation by sampling valve (No. 5 in Fig. 1) according to the assumed time schedule of our experiment, i.e. $0,2,4,6,8,12,24,48,72,96,120,147 \mathrm{hr}$, to $40 \mathrm{~mL}$ glass vials. Then, the samples were extracted to the stationary phase and the obtained extracts were treated by the previously described GC-MS method [15]. Prepared water samples were extracted to the stationary phase (Arias et al. 2009), by application of blue SPME fibre PDMS/DVB (polydimethylsiloxane/divinylbenzene) stationary phase film of a thickness of $65 \mu \mathrm{m}$ during $40 \mathrm{~min}$, by the headspace method at a temperature of $25^{\circ} \mathrm{C}$. 


\subsubsection{BHT oxidation in water}

The qualitative analysis of BHT oxidation products in water was performed by the method of liquid-liquid extraction and extract analysis on the GC-MS [15]. The water solution of BHT at concentration equal to $180 \mu \mathrm{g} \mathrm{BHT} / \mathrm{dm}^{3}$ was prepared in a laboratory flask of volume $1 \mathrm{dm}^{3}$. Deionized water from Milli-Q (Millipore, Germany) and methanol solution of BHT were used. Then, $5 \mathrm{dm}^{3}$ of technical oxygen was transferred through the solution with a flow rate equal to $1.3 \mathrm{dm}^{3} \mathrm{~min}^{-1}$. At the initial phase of the experiment, the content of dissolved oxygen in water, measured by BOD probe (Fisher Scientific) coupled with PSD (6500 Cyberscan, Eutech Instruments) was equal to $11.6 \mathrm{mg} \cdot \mathrm{dm}^{-3}$. A volume of $20 \mathrm{~cm}^{3}$ of BHT solution was sampled from the flask every hour of the experiment, then sampled water was a subject of extraction and GC-MS measurements, allowing identification of the organic compounds produced and measurements to be made of BHT concentration changes [15].

The order of reaction and the value of reaction rate for BHT oxidation in water were determined basing on the observed changes of BHT concentration in the tested solution, and using the available literature formulas [16].

\subsection{Modelling studies}

The numerical modelling of BHT transport in water was performed in the commercial CFD-based software Fluent (Ansys Inc.). The prepared three-dimensional modelled domain, reflecting the shape of the laboratory setup presented in Fig. 1, was consisting of 149783 finite elements and 179090 nodes (see Fig. 2). The flow of the whole water body with constant flow velocity was achieved in the model by assigning the constant value of axial velocity to the selected small volume of fluid, transferring the movement momentum to the rest of the model domain $[6,7]$.

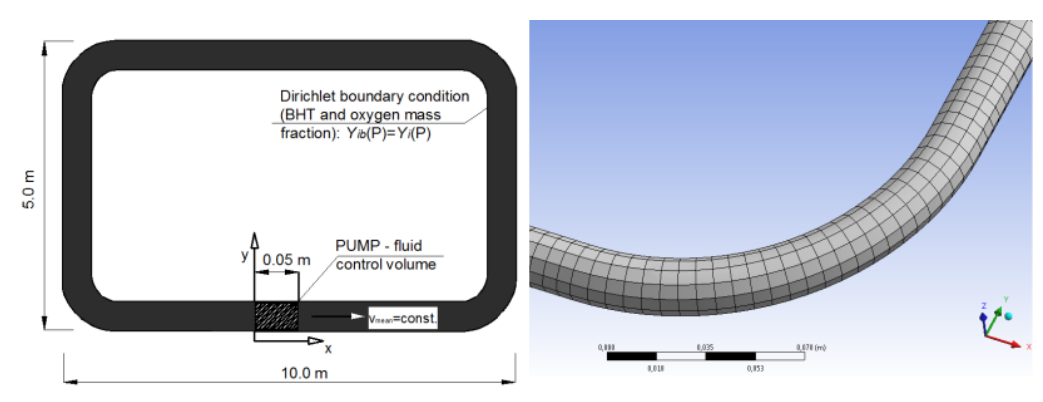

Fig. 2. Part of the finite elements' mesh and scheme of the developed model.

The performed numerical simulations covered BHT leaching to water and transport, supported by application of the reaction of BHT oxidation to BHT-OH for laminar and turbulent flow.

The efficiency of the developed model and the applied calibration procedure were validated by analysis of $\mathrm{R}^{2}$ for $\mathrm{p}=0.05$ and the following error indicators: Root Square Mean Error (RSME), Normalized Root Square Mean Error (NRSME), relation of RSME to standard deviation of observed values (RSR) and Nash-Sutcliffe coefficient (E).

The following assumptions were accepted for modelling of BHT transport in water flowing inside the closed-loop pipeline: i) time duration of modelling equal to real time of the laboratory experiment (see Tab. 1); ii) two-equations model of turbulences $\mathrm{k}-\varepsilon$ (where $\mathrm{k}$ is turbulent kinetic energy and $\varepsilon$ energy dissipation) [17]; iii) time step length equal to $60 \mathrm{~s}$; and iv) coefficient of BHT diffusion to water from pipe material, calculated according to the algorithm presented by 
Ortiz-Vazqueza et al. [18] and Widomski et al. [7], based on the results of laboratory measurements, equal to $2.9 \cdot 10^{-16} \mathrm{~m}^{2} \cdot \mathrm{s}^{-1}$ and $3.1 \cdot 10^{-16} \mathrm{~m}^{2} \cdot \mathrm{s}^{-1}$ for laminar and turbulent flow, respectively. The reaction occurring in the water body influenced by turbulences of flow (eddydissipation concept, EDC) was assumed to simulate the oxidation of BHT [19].

The input data, initial and boundary conditions assumed for the modelling are: water temperature $-296.0 \mathrm{~K}$, water viscosity $-0.001308 \mathrm{~Pa} \cdot \mathrm{s}$, BHT mole mass $-220.35 \mathrm{~g} \cdot \mathrm{mol}^{-1}$, BHT-OH mole mass - $240.35 \mathrm{~g} \cdot \mathrm{mol}^{-1}$, oxygen diffusion coefficient in water $2.5 \cdot 10^{-09} \mathrm{~m}^{2} \cdot \mathrm{s}^{-1}$, oxygen mass fraction $-1 \cdot 10^{-09}$. The values of applied Dirichlet type (I type) boundary condition for species transport were determined empirically basing on measurements of BHT concentration in water and literature reports [6-8, 19] as the dimensionless mass fraction of modelled species, equal to $9.37 \cdot 10^{-07}$ and $7.86 \cdot 10^{-07}$ for applied types of flow, respectively. Additionally, the obtained values were corrected in the model calibration process by the standard trial and error method e.g. [20, 21]. There was also assumed lack of BHT-OH migration from pipe material.

\section{Results}

\subsection{Homogeneity of BHT in PE-HD pipe material}

The measured values of BHT content in the subsequent layers of cross-section of tested pipe material showed the non-uniform distribution of BHT, which suggests the lack of homogeneity of the tested antioxidant in the polymer matrix. Fig. 3 presents the BHT content measured in subsequent layers of the tested pipe material. The various values of antioxidant content in the same layer for different pipes and various relations of BHT content in layers of the same pipe sample are clearly visible.
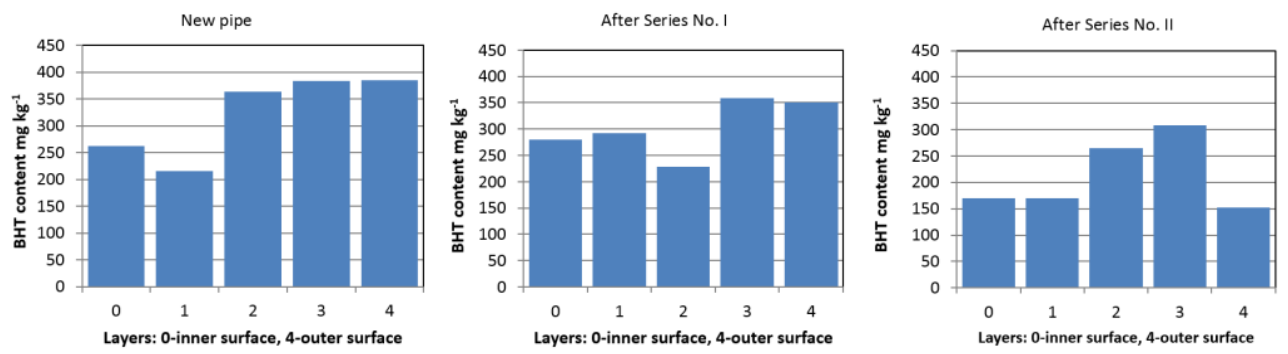

Fig. 3. Results of BHT content measurement in four samples of pipe material.

The value of mean BHT content obtained for a new pipe equal to $348.2 \pm 20.22 \mathrm{mg} \cdot \mathrm{kg}^{-1}$ was then applied to the determination of diffusion coefficient for BHT migration to water.

\subsection{Measurements of BHT leaching to water}

The time-variable concentrations BHT measured in water sampled from the laboratory setup for two tested variants of flow velocity and according to the assumed time schedule are presented in Fig. 5, which shows that in all tested cases the migration of BHT into flowing water was observed. For the initial time step of our laboratory measurements, the following initial concentrations of BHT were observed: 0.0558 , and $0.022 \mathrm{mg} \cdot \mathrm{dm}^{-3}$ for Series No. I and II, respectively. The presented initial concentrations of studied antioxidant result from the time duration between the filling of the laboratory installation with test water and the beginning of sampling. This period of time, not exceeding $60 \mathrm{~min}$, is due to the required preparatory operations necessary to operate the laboratory installation, e.g., filling with water, venting and flow velocity adjustments. 
The maximum measured concentrations of BHT in water were 0.190 and $0.145 \mathrm{mg} \cdot \mathrm{dm}^{-3}$ for laminar and turbulent flow, respectively. However, taking into account the previously described initial values of BHT concentration for the beginning of our experiment (the first sampling), the observed increase and difference between the final and initial concentrations were 0.134 and $0.123 \mathrm{mg} \cdot \mathrm{dm}^{-3}$, respectively. The lowest, more than threefold, increase of BHT concentration in relation to its initial value was observed during the laboratory experiment for laminar flow of water inside the applied closed-loop pipeline. The values of increase of tested oxidant concentration for turbulent flow were higher, approx. six times. Our observations are in agreement with the studies reported by Denberg et al. [3] suggesting that turbulences of flow or sudden changes of fluid velocity flow trigger diffusion of pollutants from the surface of pipe material to water, and cause the faster mixing of pollutants inside the water stream, thus increasing their concentration in turbulent flows.

\subsection{Oxidation of BHT in water}

Three products of BHT degradation, the results of BHT oxidation, were identified in water: 2,6-di-t-butyl-4-(3-oxopropyl) phenol, 2,4,6-tri-sec-butyl phenol and 3,5-di-t-butyl-4hydroxybenzoic acid. The first product of oxidation, BHT-OH, was included in further researches. Based on the obtained results for the qualitative identification and reported possible pathways of 3,5-di-t-butyl-4-hydroxy-toluene [22], the possible reaction of BHT oxidation in water was presented in Fig. 4.

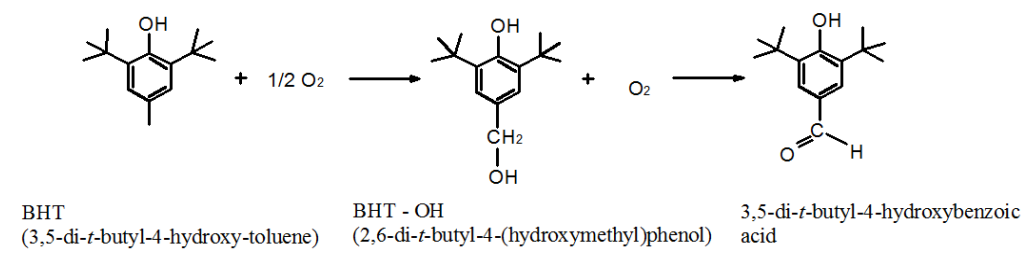

Fig. 4. The possible reaction of BHT oxidation in water.

The calculated first order reaction rate constant, based on observed changes of BHT concentration in water and literature formulas [16], is equal to $\mathrm{k}=0.0116 \mathrm{~min}^{-1}$.

\subsection{Numerical simulations}

Numerical calculations of BHT transport in water were performed for two velocities of water flow applied during the laboratory test and for the same time duration. Additionally, the reaction of chemical oxidation of BHT to BHT-OH in water was included in our simulations. The results of our calculations covered spatial distribution of BHT and BHT-OH mass fraction in water for each calculated time step.

Fig. 5 presents changes of mean BHT concentration in the water body obtained during laboratory measurements and numerical calculations for different velocity and Reynolds number of water flow, and changes in calculated BHT-OH concentration.

The obtained shapes of the curves representing time-variable calculated BHT concentration with the included reaction of BHT oxidation generally reflect the shapes of the curves representing values measured during our laboratory tests for the same flow conditions. Maximum calculated concentrations of BHT presented in Fig. 5 were equal to 0.182 and $0.168 \mathrm{mg} \cdot \mathrm{dm}^{-3}$ for laminar and turbulent flow, respectively. The obtained error indicators and regression characteristics were at the level of RSME $=0.020-0.022 \mathrm{mg} \cdot \mathrm{dm}^{-3}$, 
NRSME $=0.150-0.179$, RSR $=0.49-0.50$, Nash-Sutcliffe coefficient $E=0.723-0.739$ and $\mathrm{R}^{2}=0.901-0.984$ for $\mathrm{p}=0.05$.

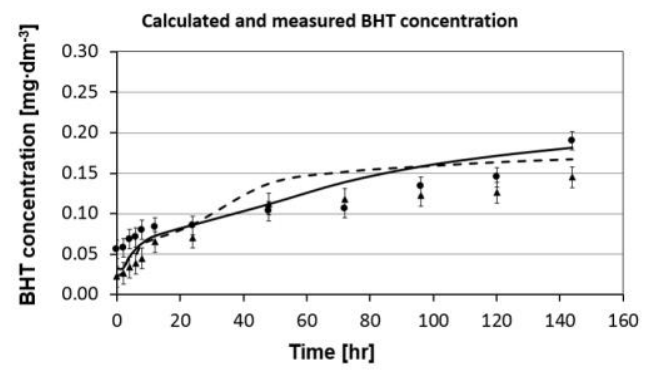

- measured, laminar flow, $\mathrm{v}=0.08 \mathrm{~m} / \mathrm{s}$ ^ measured, turbulent flow, $\mathrm{v}=2.5 \mathrm{~m} / \mathrm{s}$

—calculated, laminar flow, $v=0.08 \mathrm{~m} / \mathrm{s}--$ calculated, turbulent flow, $v=2.5 \mathrm{~m} / \mathrm{s}$

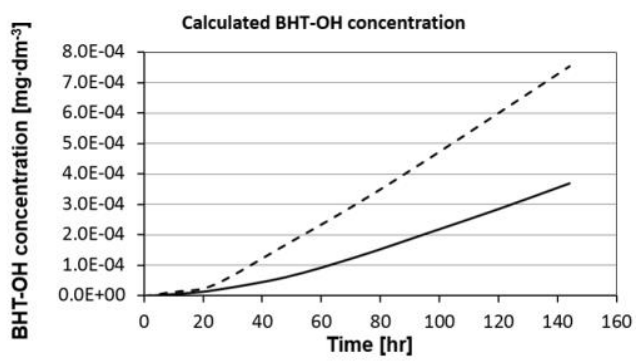

— laminar flow, v=0.08 m/s - - turbulent flow, $v=2.5 \mathrm{~m} / \mathrm{s}$

Fig. 5. Calculated and measured values of BHT concentration in water and changes in calculated BHT-OH concentration.

The modelled product of BHT degradation appeared in water during the second hour of duration of each variant of simulation - see Fig. 5. The highest calculated mean concentration of BHT-OH equal to $0.91 \cdot 10^{-3} \mathrm{mg} \cdot \mathrm{dm}^{-3}$ was noted for the turbulent flow. The presented value of BHT-OH concentration is approx. 50\% higher than that calculated for laminar flow. These results may explain the lower increase of BHT content in the case of turbulent flow noted during our laboratory experiment.

\section{Summary}

Results of BHT content measurements in the applied pipe material showed noticeable but spatially non-uniform content of the tested pollutant in the sampled material. Thus, the homogeneity of tested antioxidant in the polymer matrix of the standard pipe, easily available on the market, was not observed. Laboratory measurements of BHT concentration during the studies of BHT migration to water performed for the dynamic flow conditions, with two applied velocities of flow, showed significant increase of BHT concentration in water, resulting from migration of the tested antioxidant to water during approx. six days of experiment. The relation between conditions of flow and antioxidant migration rate, as well as its maximum observed mean concentration, was observed.

Our numerical simulations showed that rate of BHT degradation to BHT-OH is related to type of flow, the highest calculated concentration of a product of antioxidant degradation was observed for turbulent flow, the lowest for the laminar. The obtained fits of our numerical results to the measured data, with the reaction of BHT degradation to BHT-OH included, were better than the fits previously reported by Widomski et al. [6,7]. The calculated values of RSME, NRSME, RSR, E and $\mathrm{R}^{2}$ showed that our model proves the acceptable calibration for the applied cases. However, there are several sources of uncertainty which may affect the predicting capabilities of the developed model and the quality of the obtained results. The possible sources of uncertainty in our modelling attempt were: i) assumed type of turbulence reaction; ii) several input data, especially value of BHT diffusion coefficient to water; iii) assumed value of boundary condition for BHT transport based on laboratory measurements and model calibration; and iv) assigned type, order and rate constant of BHT to BHT-OH oxidation reaction.

The great diversity of available polyethylene pipes, variable added amounts of technological additives and possibly their lack of uniform spatial distribution in the polymer structure may result in the fact that the BHT concentrations observed for other 
pipelines may differ from the measured and calculated values reported in our studies. For this reason, it was necessary to use the modelling input data based on laboratory measurements for a specific material used in the tests. We are aware that such an approach may significantly limit the universality of the developed model.

\section{References}

1. W.A. Adams, Y. Xu, J.C. Little, A.F. Fristachi, G.E. Rice, C.A. Impellitteri, Environ. Sci. Technol. 45, 16, 6902-6907 (2011)

2. C. Anselme, K. Nguyen, A. Bruchet, J. Mallevialle, Environ. Technol. Lett. 6, 477-488 (1985)

3. Ansys Inc. Ansys Fluent UDF Manual (2009)

4. M. Bertoldo, F.Ciardelli, Polymer 45, 26, 8751-8759 (2004)

5. M. Denberg, E. Arvin, O. Hassager, J. Water Supply Res. T. 56, 6-7, 435-443 (2007)

6. C. Devilliers, B. Fayolle, L. Laiarinandrasana, S. Oberki, E. Gaudichet-Maurin, Polym. Degrad. Stab. 96, 7, 1361-1368 (2011)

7. E. Fries, W. Püttmann, Water Research 36, 9, 2319-2327 (2002)

8. P.G. Gandek, T.A. Hatton, R.C. Reid, Ind. Eng. Chem. Res. 28, 7, 1036-1045 (1989)

9. K. Han, T. Chu, Reaction Rate Constant Computations: Theories and Applications (RSC Publishing, Hardcover, 2013)

10. B. Kowalska, D. Kowalski, A. Rożej, J. Water Supply Res. T. 60, 3, 137-146 (2011)

11. B.E. Launder, D.B. Spalding, Letters in Heat and Mass Transfer 1, 2, 131-138 (1974)

12. S.X. Liu, M. Peng, L. Vaneb, Chem. Eng. Sci. 59, 24, 5853-5857 (2004)

13. M. Matsuo, K. Mihara, M. Okuno, H. Ohkawa, J. Miyamoto, Food Chem. Toxicol. 22, 5, 345-354 (1984)

14. N. Mikami, H. Gomi, J. Miyamoto, Chemosphere 8, 5, 305-310 (1979)

15. A. Musz-Pomorska, B. Kowalska, Ecol Chem Eng S. 2, 22, 219-229 (2015)

16. H. Ortiz-Vazquez, J. Shin, H. Soto-Valdez, R. Auras, Polym. Test. 30, 5, 463-471 (2011)

17. PN-EN 12201-2: 2011 Plastics piping systems for water supply, and for drainage and sewerage under pressure - Polyethylene (PE), Part 2: Pipes.

18. I. Skjevrak, A. Due, K.O. Gjerstad, H. Herikstad, Water Research 37, 8, 1912-1920 (2003)

19. M. Telenta, J. Duhovnik, F. Kosel, V.J. Šajn, Wind Eng. Ind. Aerodyn. 124, 99-108 (2014)

20. P. Tomboulian, L. Schweitzer, K. Mullin, J. Wilson, D. Khiari, Water Sci. Technol. 49, 9, 219-226 (2004)

21. M.K. Widomski, B. Kowalska, D. Kowalski, Ochrona Środowiska 34, 3, 33-37 (2012)

22. M.K. Widomski, B. Kowalska, D. Kowalski, M. Kwietniewski, J. Czerwiński, In: S. Mambretti, C.A. Brebbia (eds.), Urban Water (WIT Press, 2012)

23. J.M. Zhang, H.P. Lee, B.C. Khoo, C.P. Teo, N. Haja, K.Q. Peng, J. Environ. Eng. 137, 7, 575-584 (2011) 